\title{
Listening to Mothers: What's Helpful for Mothers Experiencing Perinatal Depression
}

Approximately 1 in 8 women experience perinatal depression, a depressive episode that occurs during pregnancy or within the first year after delivery or adoption. The perinatal period is an ideal time to screen, diagnose, and treat depression as women have frequent and regular contact with health care providers (Smith et al., 2009; Weissman et al., 2006). Current screening efforts may improve detection of perinatal depression (Birndorf, Madden, Portera, \& Leon, 2001) but they do not improve treatment entry or outcomes (Gilbody, Sheldon, \& House, 2008; Kozhimannil, Adams, Soumerai, Busch, \& Huskamp, 2011; Smith et al., 2009). Understanding the experiences of women who have experienced perinatal depression may help inform needed changes in how health care professionals and organizations screen, diagnose, and treat perinatal depression.

The following recommendations come from 27 mothers who shared their own experiences of perinatal depression. See Overcoming Barriers to Addressing Perinatal Depression: Perspectives of Women for study details.

\section{What Providers Can Do to Help}

- Have open lines of communication between providers (e.g., primary care, OB/Gyn and psychiatric providers) to better coordinate and facilitate mental health care for mothers.

- Be knowledgeable about treatment options including medication and non-medication treatments.

- Prepare women for the emotional spectrum of pregnancy and the postpartum period by providing psychoeducation about the symptoms of perinatal depression and challenges that may occur.

- Provide opportunities for mothers to process the birth experience.

- Create a conversation about perinatal depression - have literature available on perinatal depression and discuss it with mothers.

- Ask mothers specific questions to learn about their mental health - e.g., How are you sleeping? How are you eating? Are you crying during the day - if yes, how often? How are you coping with having a new baby?

\section{Community Resources that Help}

..I did find support through the hospital. They have a new mother's group and that was extremely helpful, just talking to other mothers and meeting with other people and... That was definitely the most, the most support I got."
- Supportive, sympathetic and helpful family members and providers, (e.g., supportive partner and/or parent, lactation consultant).

- A safe environment to discuss the struggles and challenges of motherhood (e.g., community programs designed specifically to support new mothers).

- Concrete strategies to help alleviate stress and anxiety associated with motherhood (e.g., finding someone to watch the baby so mothers can have some alone time, taking an exercise class).

Take Home Message: Mothers who have experienced perinatal depression are quite clear about what supports and resources are most helpful to them. Providers and community members should listen to their wisdom. 